\title{
Quaternary ammonium isobaric labeling for a relative and absolute quantification of peptides
}

Mateusz Waliczek, Bartosz Setner, Monika Kijewska, Remigiusz Bąchor, Dorota Gaszczyk, Piotr Stefanowicz, Zbigniew Sze wczuk

Faculty of Chemistry, Wrocław University, Poland

https://doi.org/10.17952/35EPS.2018.041

Isobaric tags for relative and absolute quantitation (iTRAQ) has been a labeling method used in quantitative analysis of peptides by tandem mass spectrometry. This technique allow to determine the amount of peptides in complex mixtures in a single experiment. Isobaric tag usually consists of three functional groups: a chemically reactive group (active ester based on N-hydroxysuccinimide group), an isotopically modified balance group and a reporter group, which is the third part of the tag. The proper isotopic substitution in the balance and reporter group results in formation of isobaric tags, which have identical molecular weight.

The conventional iTRAQ workflow relies on simultaneous digestion and labeling of a few samples (e.g. 12-plex). Finally, the samples are pooled and analyzed by LC-MS. During MS/MS experiment, the reporter ions of differential masses are released from peptide to give sample-specific quantitation of a particular peptide.

Although the isobaric labeling quantification has become a widely used method in proteomics studies, the currently available isobaric tags are characterized by relatively high price and do not offer significant improvement of detection limit. Tagging of peptides with quaternary ammonium salt (QAS) has been widely used technique allowing to increase sensitivity of detection by ESI-MS[1]. Recently, we proposed a linear[2] and bicyclic[3] QAS as ionization enhancers for analysis of peptides at the attomole level[4].

Herein, we proposed two novel approaches, representing new classes of quaternary ammonium isobaric tags for relative and absolute quantitation(QA-iTRAQ 2-plex)[5]. The first procedure is based on application of $\mathrm{N}$-(4-iodobenzylpiperidinylcarbonyl)glycine tag containing two deuterium atoms incorporated into the benzyl group (characterized by high stability) or glycine (balance group) residue (Fig. 1).

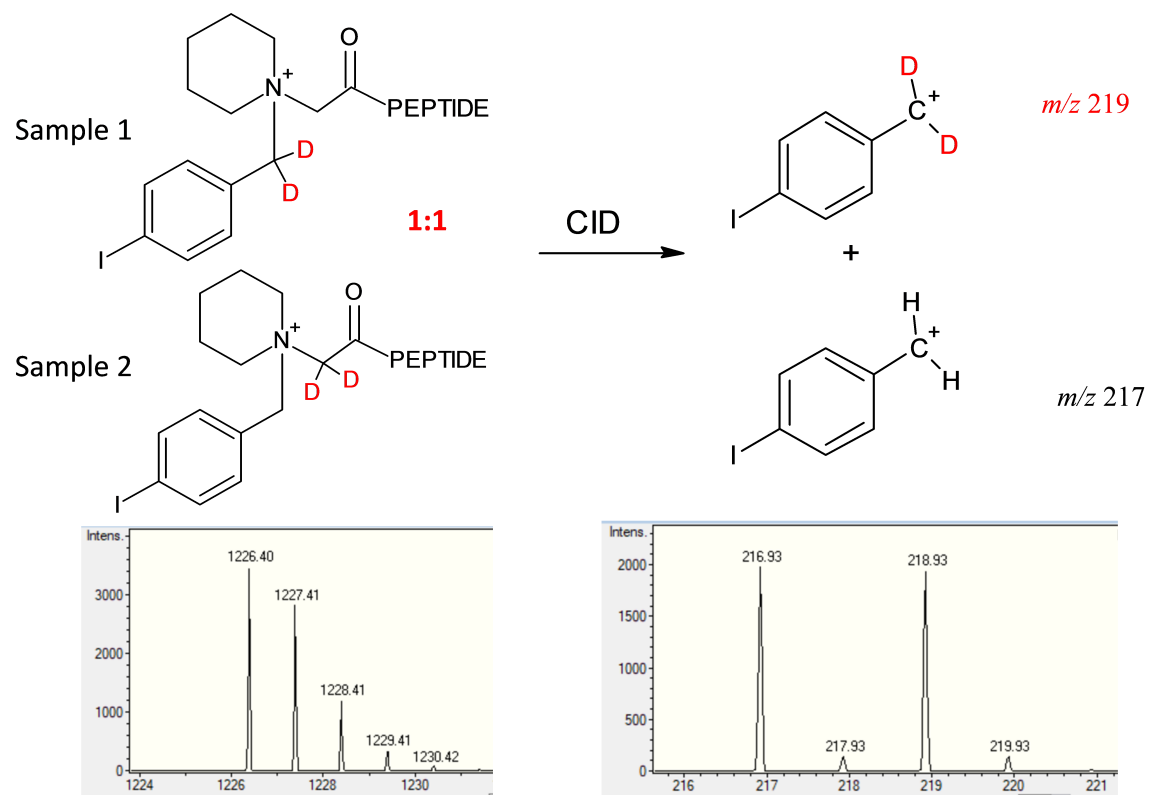

Figure 1: MS spectrum of the isobars and formation of reporter ions at $\mathrm{m} / \mathrm{z} 219$ and 217 after CID fragmentation.

The second approach is based on the combination of enzymatic ${ }^{16} \mathrm{O} /{ }^{18} \mathrm{O}$ exchange followed by chemical modification of C-terminal lysine side chain with isotopically labeled pyrylium salt[6], which in turn allows for isobaric labeling of tryptic digests (Fig. 2). Each of modified peptide contain zero or four ${ }^{13} \mathrm{C}$ atoms in the reporter group, which results in a unique reporter mass during tandem MS/MS for sample identification and relative quantitation. 


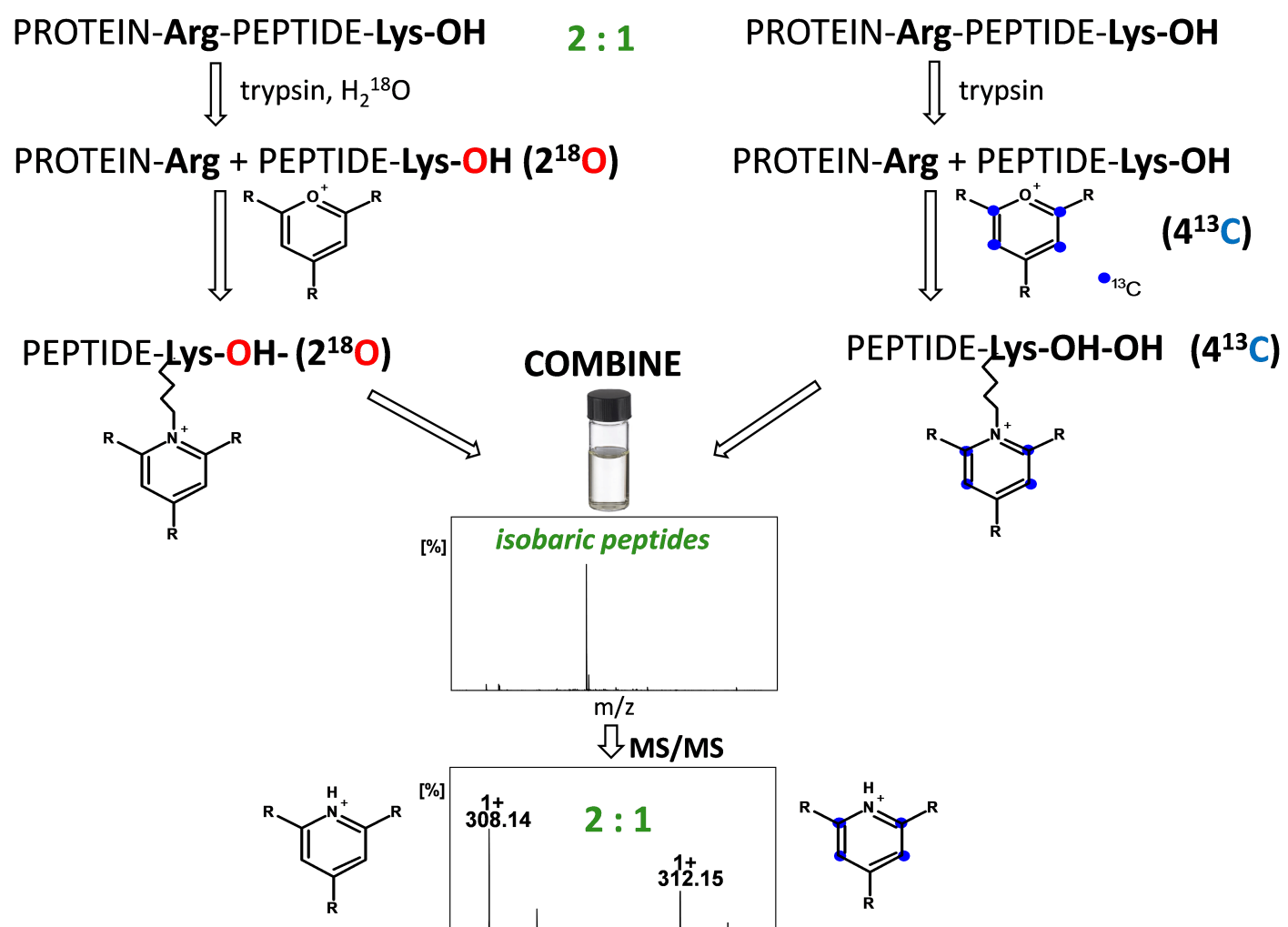

Figure 2: Scheme presenting the procedure of duplex formation.

We tested two different types of QAS allowing for formation of 2-plex on synthetic peptides, protein tryptic digests as well as on a more complex sample - podocyte cells digest. The obtained results suggest usefulness of the isobaric ionization tags for relative and absolute quantification of trace amounts of peptides.[7]

This work was supported by the National Science Centre in Poland grant [UMO-2016/23/B/ST4/01036]

\section{References}

[1] P. Stefanowicz, A. Kluczyk, Z. Szewczuk, Amino Acids, Pept. Proteins, 2016, 40, 36.

[2] M. Cydzik, M. Rudowska, P. Stefanowicz, Z. Szewczuk, J. Am. Soc. Mass Spectrom., 2011, 22, 2103.

[3] B. Setner, M. Rudowska, E. Klem, M. Cebrat, Z. Szewczuk, J. Mass Spectrom., 2014, 49, 995.

[4] R. Bąchor, P. Mielczarek, M. Rudowska, J. Silberring, Z. Sze wczuk, Int. J. Mass Spectrom., 2014, 362, 32.

[5] B. Setner, P. Stefanowicz, Z. Szewczuk, J. Mass Spectrom., 2018, 53, 115.

[6] M. Waliczek, M. Kijewska, M. Rudowska, B. Setner, P. Stefanowicz, Z. Szewczuk, Sci. Rep., 2016, 6, 37720.

[7] M. Waliczek, R. Bąchor, M. Kijewska, D. Gąszczyk, K. Panek-Laszczyńska, A. Konieczny, K. Dąbrowska,

W. Witkiewicz, K. Marek-Bukowiec, J. Tracz, M. Łuczak, P. Stefanowicz, Z. Szewczuk, Anal. Chim. Acta, 2018, in press: doi.org/10.1016/j.aca.2018.10.012 